\title{
A PARALLEL 3D MODEL FOR THE MULTI-SPECIES LOW ENERGY BEAM TRANSPORT SYSTEM OF THE RIA PROTOTYPE ECR ION SOURCE VENUS*
}

\begin{abstract}
The driver linac of the proposed Rare Isotope Accelerator (RIA) requires a great variety of high intensity, high charge state ion beams. In order to design and to optimize the low energy beam line optics of the RIA front end, we have developed a new parallel three-dimensional model to simulate the low energy, multi-species ion beam formation and transport from the ECR ion source extraction region to the focal plane of the analyzing magnet. A multisection overlapped computational domain has been used to break the original transport system into a number of subsystems. Within each subsystem, macro-particle tracking is used to obtain the charge density distribution in this subdomain. The three-dimensional Poisson equation is solved within the subdomain and particle tracking is repeated until the solution converges. Two new Poisson solvers based on a combination of the spectral method and the multigrid method have been developed to solve the Poisson equation in cylindrical coordinates for the beam extraction region and in the Frenet-Serret coordinates for the bending magnet region. Some test examples and initial applications will also be presented.
\end{abstract}

\section{INTRODUCTION}

The RIA linac driver requires a great variety of high charge state, high intensity ion beams from the Electron Cyclotron Resonance (ECR) ion sources. This puts a strong challenge not only for the design of ECR ion sources but also for the design of low energy beam transport (LEBT) systems. Computational tools help to explore wide range of parameter space, to identify the particle loss condition, and to optimize the system design and operation. Previous design of the LEBT depended on a twodimensional envelope model such as TRACE-2D [1, 2], or a time dependent bunched beam simulation with periodic boundary condition [3] or a simplified model with infinite beam pipe length [4]. A fully three-dimensional self-consistent simulation of multiple charge state ion beam transport in the LEBT will help to minimize the particle losses in such a system. Moreover, using the new Poisson solvers developed here and an implementation on high performance parallel computers, we can run the simulation with a large number of macroparticles and still have a quick return time.

\footnotetext{
${ }^{*}$ Work supported by the Director, Offi ce of Energy Research, Offi ce of High Energy and Nuclear Physics, Nuclear Physics Division of the U.S. DOE under Contract DE AC03-76SF00098.

$\dagger$ jqiang@1bl.gov
}

The low energy ion beam transport inside the LEBT is different from the beam transport inside the RF linac. Inside the RF linac, the beam is longitudinally bunched to a few millimeters by the time dependent RF field. Inside the LEBT, there is no longitudinal bunching, the particles extend longitudinally through the whole system to form a continuous beam. The length of beam could be from a few meters to ten meters. To model the particle transport in such a system, we need to solve the time-independent Vlasov-Poisson equations including transverse boundary conditions. A brutal force approach is to model the whole system as one computational domain directly. However, this is computationally impractical if a good numerical precision is required. A very high numerical resolution is needed in order to accurately model the plasma surface at the exit of ECR ion source. On the other hand, this is also not necessary since the particles at the beginning may not affect the particles near the end due to the large longitudinal to transverse aspect ratio and the shielding of transverse conducting wall. Hence, we can divided the whole beam into multiple overlapped segments. For each segment, we solve a time-independent Vlasov-Poisson equations with Dirichlet boundary conditions on the left end and Neumann boundary conditions on the right end. The left end of the segment is chosen inside the domain of preceding segment so that the potential and the density distribution obtained in the preceding segment are used as the left boundary conditions of this segment. For each segment, an iterative particle-tracking method has bean used to obtain the converged solution of the time-independent Vlasov-Poisson equations. Here, we have used a 2nd order leap-frog algorithm to solve the Lorentz equation for each particle subject to the external field and the space-charge force. The particles are deposited onto grid to obtain the charge density distribution on the mesh. After the particles have passed through the local domain, the Poisson equation is solved. The new electric potential is used to calculate the space-charge field and the particle tracking is redone using this new field. This procedure is repeated for a number of times until the potential change is below some specified error level and the particle trajectories converge.

\section{Solution of the Poisson Equation in Cylindrical Coordinates}

A major section of the LEBT system, such as extraction region and solenoid focusing region, has a geometry of 
round shape with azimuthal symmetry. For such a system, we can write the Poisson equation in cylindric coordinates as:

$$
\frac{\partial^{2} \phi}{\partial r^{2}}+\frac{1}{r} \frac{\partial \phi}{\partial r}+\frac{1}{r^{2}} \frac{\partial^{2} \phi}{\partial \theta^{2}}+\frac{\partial^{2} \phi}{\partial z^{2}}=-\rho / \epsilon_{0}
$$

Here, $\phi$ denotes the electrostatic potential, $\rho$ the charge density function, $r$ and $z$ the radial and longitudinal distance. Since both the electric potential and the charge density are periodic function of $\theta$, we can approximate the potential $\phi$ and source term $\rho$ as:

$$
\begin{aligned}
& \rho(r, \theta, z)=\sum_{m=-N_{m} / 2}^{N_{m} / 2-1} \rho^{m}(r, z) \exp (-i m \theta) \\
& \phi(r, \theta, z)=\sum_{m=-N_{m} / 2}^{N_{m} / 2-1} \phi^{m}(r, z) \exp (-i m \theta)
\end{aligned}
$$

Substituting equations 2 and 3 into the original Poisson equation 1, we obtain a group of decoupled twodimensional partial differential equations in $(r, z)$ as:

$$
\frac{\partial^{2} \phi^{m}}{\partial r^{2}}+\frac{1}{r} \frac{\partial \phi^{m}}{\partial r}-\frac{m^{2}}{r^{2}} \phi^{m}+\frac{\partial^{2} \phi^{m}}{\partial z^{2}}=-\rho^{m} / \epsilon_{0}
$$

The boundary conditions at the radial edge are assumed as Dirichlet boundary conditions with given potential from the conducting wall or as Neumann boundary conditions if there is no conducting wall. The above equations are solved using a finite difference multigrid method.

The multigrid method is a method to solve linear and nonlinear algebraic equations using multiple grids with different discretization resolution on each grid. By changing the resolution of the discretization, the low frequency errors in the residues of the iteration can be removed on a coarser grid, while the high frequency errors can be resolved on a fine grid. The computational cost of this method scales linearly with the number of grid points and the convergence rate will not degrade with finer mesh size.

The above solver is first tested with a uniform round beam inside a conducting pipe. Here, we have used a Neumann boundary conditions at both ends. The numerical solution together with the analytical solution is given in Fig. 1. We see that the agreement between the numeri-

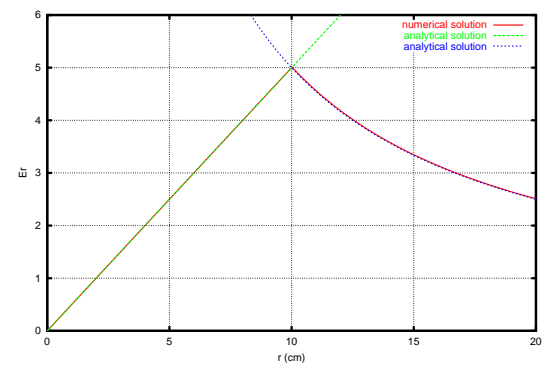

Figure 1: Radial electric field from the numerical solution and from the analytical solution.

cal solution and the analytical solution is very good. As a second test, we calculated the potential inside the extraction region with a $20 \mathrm{KV}$ plasma electrode, a $-1 \mathrm{kV}$ puller electrode, and a $0 \mathrm{KV}$ ground electrode. The electric potential on the axis as a function of distance is given in Fig. 2 together with the solution using another code WARP [6]. It is seen that the two solutions agree with each other very

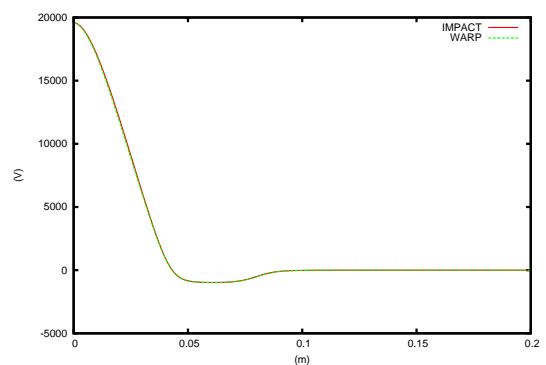

Figure 2: Electric potential on the axis from the new solver and from the WARP solver.

well.

\section{Solution of the Poisson Equation in Frenet-Serret Coordinates}

The preceding Poisson solver is applicable for a round system with azimuthally symmetric geometry. For some element in the LEBT, such as bending magnet, the system has a rectangular cross-section with different horizontal bending angles. The bending magnet provides not only a transverse focusing of the ion beam but also a selection of different charge states for further transport. In this element, we will use a Frenet-Serret coordinate since this coordinate reduces to the normal Cartesian coordinate when the curvature is zero. For perfect conducting plates, the electric potential will vanish on vertical walls. We can approximate the potential and the charge density distribution as:

$$
\begin{aligned}
\rho(x, y, z) & =\sum_{m=1}^{N_{m}} \rho^{m}(x, z) \sin (m \pi y / a) \\
\phi(x, y, z) & =\sum_{m=1}^{N_{m}} \phi^{m}(x, z) \sin (m \pi y / a)
\end{aligned}
$$

where $a$ is the full vertical aperture width. Substituting these equations into the Poisson equation and using the orthogonal condition of sine functions, we obtain a group of decoupled two-dimensional partial differential equations:

$$
\begin{aligned}
\frac{1}{1+h x}\left(\frac{\partial}{\partial x}(1+h x) \frac{\partial \phi^{m}}{\partial x}+\frac{\partial}{\partial z}\right. & \left.\frac{1}{1+h x} \frac{\partial \phi^{m}}{\partial z}\right)- \\
\frac{m^{2} \pi^{2}}{a^{2}} \phi^{m} & =-\rho^{m}(x, z) / \epsilon_{0}
\end{aligned}
$$

The resulting two-dimensional partial differential equations are solved using the same finite difference multigrid method described before.

As a test of this Poisson solver, we calculate the electric field inside a bended conducting pipe with a longitudinal uniform and transverse Gaussian distribution beam and 
different bending curvatures. Fig. 3 shows the horizontal electric field as a function of $y$ at $x=0$ for different bending curvatures. We see that as the curvature approaches to

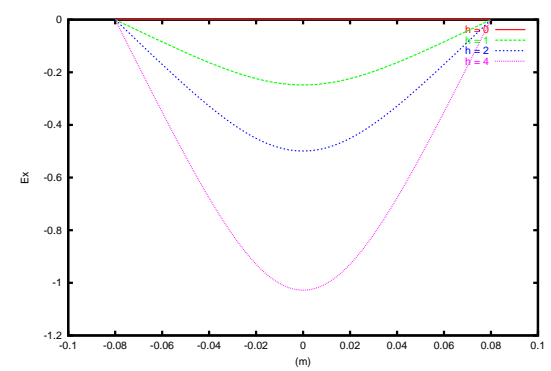

Figure 3: Horizontal electric field along the vertical axis with a bended rectangular pipe with different curvatures.

zero, the horizontal field vanishes due to the symmetry of the beam density distribution.

\section{APPLICATIONS}

The simulation tool developed here has been applied to the study of the ion beam transport out of an superconducting ECR ion source, VENUS, at Lawrence Berkeley National Laboratory [5]. It consists of an extraction region, a glaser solenoid focusing lens, and a bending analyzing magnet for charge selection. The total length is about 3.5 meters. As a first example, we have studied the three-dimensional space-charge effects of a $3 \mathrm{~cm}$ parallel beam passing through the bending magnet. This is done by comparing the simulation results from the threedimensional code developed here with a two-dimensional simulation results of the WARP code. Here, the WARP simulation includes only the transverse space-charge effects. The transverse rms size of the beam as a function of the distance is given in Fig. 4 from $1 \mathrm{~mA}$ current and in Fig. 5 for $10 \mathrm{~mA}$ current. We see that before the fo-

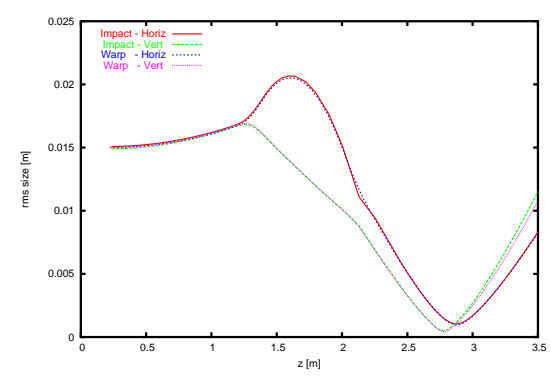

Figure 4: Transverse rms beam size as a function of distance from the $3 \mathrm{D}$ simulation and 2D simulation for a beam with $1 \mathrm{~mA}$ current.

cusing waist, both simulations agree very well. After the focusing waist, there is only small difference between the two simulations for the $1 \mathrm{~mA}$ case. This suggests that the longitudinal density variation is not significant for such a system with $1 \mathrm{~mA}$ current. A two-dimensional simulation

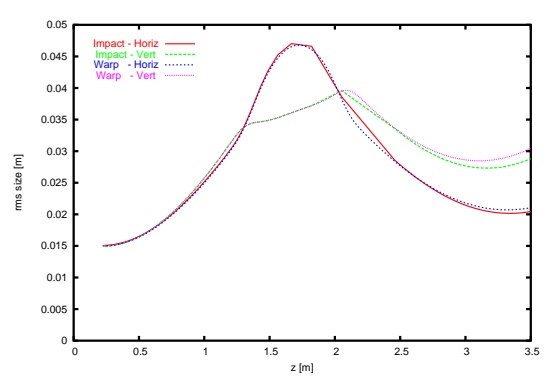

Figure 5: Transverse rms beam size as a function of distance from the 3D simulation and 2D simulation for a beam with $10 \mathrm{~mA}$ current.

might be good enough. However, with a large $10 \mathrm{~mA}$ current, the longitudinal density variation around the focusing waist is no longer small. There, we observe some differences between the three-dimensional simulation results and the two-dimensional simulation results.

As another example of applications, we have simulated a $2 \mathrm{~mA} \mathrm{H}^{+}$and $\mathrm{H}_{2}^{+}$ion beam transport through the LEBT. Here, we have used 20, 480 macroparticles and five overlapped segments in the simulation. The transverse rms size as a function of distance is given in Fig. 6. With the chosen

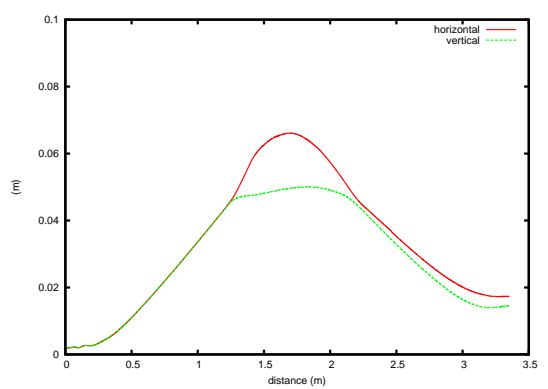

Figure 6: Transverse rms beam size as a function of the VENUS LEBT distance.

bending magnet strength for the $\mathrm{H}^{+}$, all the $\mathrm{H}_{2}^{+}$particles are lost inside the magnet.

\section{REFERENCES}

[1] K. R. Crandall, D. P. Rusthoi, LA-UR-97-886, Los Alamos National Laboratory Report, May 1997.

[2] D. Wutte, M. A. Leitner, C. M. Lyneis, C. E. Taylor and Z. Q. Xie, Proc. Eighth International Conference on Heavy-Ion Accelerator Technology, October 5-9 1998, Argonne, Illinois.

[3] Q. Zhao, et al., Proc. 16th International Workshop on ECR Ion Sources, September 26-30 2004, Bekeley, California.

[4] T. Thuillier, et al., Proc. 16th International Workshop on ECR Ion Sources, September 26-30 2004, Bekeley, California.

[5] D. Leitner, et al., Proc. 16th International Workshop on ECR Ion Sources, September 26-30 2004, Bekeley, California.

[6] A. Friedman, D. P. Grote, I. Haber, Phys. Fluids B4 (1992) 2203. 